\title{
LA CONSTRUCTION SOCIALE DU DROIT AUJOURD'HUI: ENJEUX ET MODALITÉS PERSPECTIVES ENVIRONNEMENTALES ET AU-DELÀ
}

\author{
Adelie Marie Andree Pomade*
}

\begin{abstract}
RÉSUMÉ
La fabrication du droit par la société est aujourd'hui plus qu'une hypothèse. Il s'agit d'une certitude, d'une réalité. La société inspire les cadres théoriques du droit par la revendication de ses valeurs et de sa pratique. Elle en modèle le contenu et les processus par la mise en avant de ses droits fondamentaux et de ses savoirs et expériences. Cette construction s'accélère par la rencontre de la Science et de la société ou encore par le croisement d'enjeux écologiques et économiques. Elle est ancrée dans une volonté commune de prendre son destin en main. Le droit ainsi produit entraîne une reconfiguration du rôle de l'autorité publique dans sa mission de régulation des comportements, et l'avènement de nouveaux modèles de régulation sociale et politique issus d'une réappropriation de la chose juridique par nos sociétés contemporaines.
\end{abstract}

MOTS-CLES: Théorie du droit. Réalité sociétale. Sociologie du droit. Citoyenneté environnementale.

\begin{abstract}
Today, the manufacture of Law by Society is more than a hypothesize. It is a reality. Society inspires legal theoretical framework by claiming its values and its practices. It also models legal contains and process by highlighting its fundamental rights, its knowledge and its experiences. This construction accelerates by several factors. It can be the meeting of Science and society, or the crossing of ecological and economic issues. Furthermore, this construction is established by a strong common human will of controlling its own destiny. This news perspective leads to a reconfiguration of the public authority's role in its mission of regulation of conducts. It leads to the rise of news models of
\end{abstract}

* Doutorado em Direito (Especialidade Direito Ambiental) - Université d'Orléans (2009) 
social and political regulation coming from a reappropriation of the legal thing by modern societies.

KEYWORDS: Theory of Law. Social reality. Sociology of Law. Environmental citizenship.

«La construction sociale du droit» est une expression que l'on rencontre de plus en plus fréquemment chez les juristes ${ }^{1}$ et les sociologues $^{2}$, mais elle emporte un ensemble de questionnements tendant à la fois à la justification de son emploi et à ses enjeux et modalités. Sur quoi construire le doit aujourd'hui? Pourquoi parler de construction sociale du droit aujourd'hui?

Quand on voit l'ampleur des défis écologiques, l'accroissement du phénomène migratoire, le creusement des inégalités sociales à l'échelle planétaire ${ }^{3}$, et que l'on prend acte des critiques concernant la démocratie représentative et les formes de régulation top-down ${ }^{4}$, de la multiplication des instances de décision qui associent à leur processus les représentants de la société civile, alors en effet on peut penser que la construction du droit est confrontée à de nouveaux défis, à de nouveaux enjeux pour lesquels les bases «classiques» de construction ne peuvent suffire.

Peut-être alors que l'une des réponses à cette question se trouve dans la sphère sociétale. On parle en effet de l'avènement d'une «démocratie participative» ${ }^{5}$ qui suggère l'implication directe des citoyens dans la conception des politiques publiques et du droit, d'une «éthique de la discussion» qui, selon Habermas, invite les personnes concernées par un problème à en discuter ensemble ${ }^{6}$. Le

\footnotetext{
${ }^{1}$ Beaudouin Dupret, Droit et Sciences Sociales, Paris: Armand Colin, coll. cursus sociologique, 2006.

2 Jacques Commaille, A quoi nous sert le droit?, Paris: Gallimard, coll. Folio Essais, 2015.

${ }^{3}$ UNESCO, World Social Science Report 2016. Challenging Inequalities: Pathways to a Just World, UNESCO, 2016.

${ }^{4}$ Jacques Chevallier, L'Etat post-moderne, Paris: LGDJ, coll. Droit et Société, 2014.

${ }^{5}$ Loïc Blondiaux, Le nouvel esprit de la démocratie. Actualité de la démocratie participative, Paris: Le Seuil, coll. La République des idées, 2008; Yves Sintomer, Le pouvoir au peuple, Paris: La Découverte, 2007.

${ }^{6}$ Jurgen Habermas, De l'éthique de la discussion, Paris: Flammarion, coll. Champs Essais, 2013.
} 
droit apparaît alors comme la résultante d'interventions d'acteurs multiples; il ne s'impose plus aux sociétés, mais il naît aussi de leur effervescence ${ }^{7}$.

Si l'on suit la pensée de Duguit selon laquelle on ajuste la production du droit à la réalité sociale ${ }^{8}$, alors le premier enjeu de la construction du droit serait sa mise en adéquation avec la réalité sociétale.

Toutefois, à bien regarder, cette réalité sociétale porte en elle des enjeux à la fois ciblés, globaux, évolutifs, exprimés par ce que le sociologue Alain Touraine appelle les nouveaux mouvements sociaux ${ }^{9}$. Ces enjeux couvrent de vastes champs thématiques, comme la famille, le genre, les migrations ${ }^{10}$, ils dépassent la sphère juridique mais sont fondamentaux pour guider la construction du droit. Parmi ces enjeux, on peut évoquer en premier lieu, le développement durable, entendu comme le développement économique, social et environnemental permettant de répondre aux besoins présents sans compromettre ceux des générations à venir ${ }^{11}$.

Un deuxième enjeu pouvant être évoqué est la transition écologique et sociale qui renvoie à la redéfinition des modes de production et de consommation de nos sociétés ${ }^{12}$. Ces deux enjeux sont par exemple au coeur des débats élevés dans toutes les régions du monde sur la prolongation ou non du recours à l'énergie nucléaire. En France, les politiques publiques promeuvent le recours à des énergies vertes comme le photovoltaïque ou l'éolien, mais en pratique on constate le maintien du fonctionnement des centrales nucléaires ${ }^{13}$. Au

\footnotetext{
${ }^{7}$ Jacques Commaille, A quoi nous sert le droit?, précit.

${ }^{8}$ Jacques Commaille, A quoi nous sert le droit?, précit.

9 Alain Touraine, «Les nouveaux mouvements sociaux: objet particulier ou problème central d'analyse sociologique ? », Revue française de sociologie, 1984, Vol. 25, p. 3-19.

${ }^{10}$ Erik Neveu, Sociologie des mouvements sociaux, Paris: La Découverte, coll. Repères, 2011.

${ }^{11}$ Rapport Brundtland, Notre avenir à tous, Oxford: Oxford University Press, 1987.

${ }^{12}$ Helga-Jane Scarwell, Divya Leducq, Annette Groux (dir.), Réussir la transition énergétique, Lille: Presses Universitaires Septentrion, coll. Environnement et Société, 2015.

${ }^{13}$ Dominique Grand, Christian Le Brun, Roland Vidil, «Transition énergétique et mix électrique: les énergies renouvelables peuvent-elles compenser une réduction du nucléaire ? », Revue de l'énergie, 2014, Vol. 19, p. 211-220.
} 
Canada, tandis que l'Ontario choisit de rénover ses centrales nucléaires, des groupements environnementaux comme Ecojustice réclament une prise en compte des impacts du nucléaire sur la santé et l'environnement au regard des désastres écologiques et humain comme Fukushima ${ }^{14}$.

Un troisième enjeu renvoie à la sécurité qui se décline sous les concepts de sécurité alimentaire ${ }^{15}$, sécurité civile, sécurité informationnelle. Ces enjeux font l'objet d'une attention particulière en droit, par exemple en France depuis 2015, sous la forme de décisions décrétant ou prolongeant l'Etat d'urgence ${ }^{16}$. Dans le même domaine, en Belgique, il s'agit de l'élaboration d'une loi de 2003 relative aux infractions terroristes qui a introduit, dans le code de pénal, les caractères d'une infraction terroriste ${ }^{17}$. Plus largement, les enjeux liés à la sécurité peuvent renvoyer plus largement à ce que l'on appelle la cyber sécurité, ce qui donna lieu en Europe à la directive Network and Information Security en $2016^{18}$.

Le dernier enjeu qu'il est possible de mentionner, bien qu'il en existe de nombreux autres, c'est la responsabilité perçue dans sa dimension éthique et philosophique. On peut la rapprocher ici du principe de responsabilité de Hans Jonas avec son impératif moral et inconditionnel de responsabilité des générations présentes à l'égard des générations futures ${ }^{19}$. C'est cet enjeu qui sous-tend par exemple, le principe de précaution.

$\mathrm{Au}$ regard de ces enjeux qui influent sur l'élaboration des normes juridiques nationales, régionales et internationales, il s'avère

\footnotetext{
14 Ecojustice, "What Ontario must learn from Fukushima nuclear disaster", 22 March 2013, http://www.ecojustice.ca/what-ontario-must-learn-from-fukushimanuclear-disaster/

15 François Collart Dutilleul, Le végétal et la sécurité alimentaire : approche juridique internationale, in William Dross (dir.), Le Végétal saisi par le droit, Bruxelles: Bruylant, 2012.

${ }^{16}$ Loi $^{\circ}$ 2016-1767 du 19 décembre 2016 prorogeant l'application de la loi $\mathrm{n}^{\circ} 55$ 385 du 3 avril 1955 relative à l'état d'urgence, JO du 20 décembre 2016.

${ }^{17}$ Loi du 19 décembre 2003 relative aux infractions terroristes, Moniteur belge du 29 décembre 2003, p. 61689.

18 Directive 2016/1148 du Parlement européen et du Conseil du 6 juillet 2016 concernant des mesures destinées à assurer un niveau élevé commun de sécurité des réseaux et des systèmes d'information dans l'Union, JOUE du 19 juillet 2016.

${ }^{19}$ Hans Jonas, Le Principe Responsabilité, Paris : CERF, 1990.
} 
possible de réfléchir aujourd'hui sur une construction du droit à partir de la société elle-même. La difficulté réside dans le fait qu'il ne semble pas exister, dans nos sociétés contemporaines, de catalogue des «fondements» sur lesquels construire le droit. $\mathrm{Au}$ contraire, on assisterait à la mise en place d'un réseau de "valeurs, données, méthodes », articulé autour de la relation droit / société.

C'est donc à partir de ces hypothèses que cet article sera décliné en envisageant une construction du droit sur des cadres théoriques redessinés par l'action sociétale, privilégiant ainsi une dimension théorique (I) et une construction du droit sur l'expression citoyenne, favorisant alors une dimension pratique (II). Nous allons ainsi nous apercevoir que cette construction sociale embrasse non seulement les dimensions de théorie du droit et de droit positif, mais aussi les dimensions méta-juridique et empirique, et que ces «fondements » théoriques et pratiques, mobilisés pour répondre à des enjeux globaux et construire le droit, sont eux-mêmes porteurs d'enjeux.

\section{LA CONSTRUCTION DU DROIT SUR DES CADRES THEORIQUES REDESSINES}

La construction du droit sur des cadres théoriques redessinés invite à reconsidérer la dimension méta-juridique, telles les valeurs portées par la société (A) et une dimension de théorie juridique (B).

\section{A. Les valeurs portées par la société}

Des valeurs sont réaffirmées par la société par un souci de gestion du collectif et de l'intérêt général dans un contexte ou l'individualisme est pourtant fortement présent. Pour évoquer ces valeurs et les expliciter, il est possible de partir de l'exemple de la gestion des communs.

Les communs suscitent une abondante littérature scientifique depuis notamment les travaux d'Elinor Ostrom ${ }^{20}$. Ils créent de nouvelles formes de vie citoyenne et déterminent parfois les agendas

20 Elinor Ostrom, Governing the Commons, Cambridge : Cambridge University Press, 2015. 
politiques. On les rencontre sous des termes variés: biens communs $^{21}$, choses communes ou de patrimoine commun ${ }^{22}$. Toutes ces expressions renvoient cependant à l'idée de «quelque chose qui appartient à plusieurs » pour lequel plusieurs objectifs sont fixés : garantir un usage élargi à tous ou à certaines communautés et en assurer la conservation, tant pour les générations présentes que pour les générations futures ${ }^{23}$. Aussi la définition des communs proposée par Elinor Ostrom, renvoie à trois éléments de base : une ressource, une communauté et un ensemble de droits et d'obligations qui sont définis par le collectif selon des modalités de gouvernance spécifiques $^{24}$. La ressource, ou le commun, peut être physique, comme des pâturages ou des réserves naturelles gérés par une communauté, ou immatériel(le), comme les savoirs locaux ou les logiciels libres. Parfois même, les communs peuvent recouvrir des champs plus vastes comme la santé, l'éducation, ou le climat.

Partant, quelles valeurs sont réaffirmées autour de la gestion de ces « ressources», de ces «communs», au point de pouvoir conduire également à construire le droit ? Il semble possible d'en évoquer au moins trois :

La solidarité, entendue comme le devoir moral envers autrui ${ }^{25}$. On retrouve cette valeur liée aux communs au travers des «zones de gratuité éphémères», en France, qui sont des espaces où chacun vient déposer des biens en bon état dont il ne se sert plus, et trouver des objets, le tout gratuitement. Ce n'est ni ce que l'on appelle une «brocante», ni un troc, et les relations marchandes y sont abolies. Les objets déposés n'appartiennent à personne et pourtant potentiellement à tous. Cette pratique invite à réécrire des aspects du droit de propriété et du droit des affaires.

L'émancipation, entendue dans un sens d'affranchissement, de

21 Oran R. Young, Rachel Bouyssou, «Gérer les biens communs planétaires. Réflexion sur un changement d'échelle », Critique internationale, 2000, Vol. 9, p. 147-160.

22 Judith Rochfeld, «Penser autrement la propriété : la propriété s'oppose-t-elle aux communs ? », Revue internationale de droit économique, 2014, p. 351-369.

${ }^{23}$ François Ost, Delphine Misonne, Marie-Sophie de Clippele, «Propriété et bien commun », ARSP Beiheft, 2016.

${ }^{24}$ Elinor Ostrom, Governing the Commons, précit.

25 Voir notamment: Jacques Leclercq, «Le devoir d'altruisme », Revue néoscolastique de philosophie, 1925, p. 29-60. 
sortie d'un système conventionnel pour adopter un positionnement différent. On peut donner ici plusieurs exemples. En environnement, cela renvoie à l'économie circulaire, nouveau concept dont l'objectif est se départir d'une vie linéaire des produits, de leur conception à leur a destruction, pour adopter une vie circulaire intégrant le recyclage en lieu et place de la destruction ${ }^{26}$. Cela a conduit à repenser la réglementation européenne en adoptant en 2015 un Paquet «économie circulaire » qui invite à concevoir d'une manière alternative la vie des produits de consommation ${ }^{27}$. Une autre forme d'émancipation en lien avec les communs et hors du champ de l'environnement mais extrêmement d'actualité renvoie aux logiciels libres qui apparaissent souvent comme l'exemple maître de l'émancipation immatérielle ${ }^{28}$. Les logiciels libres sont des logiciels téléchargeables sur Internet qui peuvent être utilisés, modifiés et redistribués sans restriction par la personne à qui ils ont été distribués. Ce sont par exemple les navigateurs internet comme Mozilla Firefox, ou les lecteurs de documents comme PDF Creator. Ces logiciels sont ainsi susceptibles d'être soumis à étude, critique et correction. Cela conduit à repenser le droit de propriété intellectuelle, au motif d'un accroissement de la diffusion des connaissances et de la publicisation des contenus.

Une autre valeur que l'on peut citer est la justice, que l'on rencontre très souvent déclinée sous les expressions de justice sociale ou de justice environnementale. La justice sociale est fondée, selon la conception retenue par les Nations Unies, sur l'égalité des droits pour tous les peuples et la possibilité pour tous les êtres humains sans discrimination de bénéficier du progrès économique et social partout dans le monde ${ }^{29}$. La justice environnementale quant à elle sous-tend

${ }^{26}$ Cyril Adoue, Rémi Beulque, Laetitia Carré, Julie Couteau, Quelles stratégies d'entreprise pour une économie circulaire moteur de croissance ? : Amorcer la transition, construire le modèle de demain, Paris : Institut de l'économie circulaire, 2014. 27 Communication De La Commission Au Parlement Européen, Au Conseil, Au Comité Économique Et Social Européen Et Au Comité Des Régions. Boucler la boucle - Un plan d'action de l'Union européenne en faveur de l'économie circulaire, COM(2015)614 final, 2 décembre 2015.

28 Hervé Le Crosnier, «Leçon d'émancipation: l'exemple du mouvement des logiciels libres », ATTAC association, 2009, en ligne : http://www.acrimed.org

29 Déclaration de l'OIT sur la justice sociale pour une mondialisation équitable, 10 juin 2008, Genève. 
qu'il existe des droits sur la nature pour tous et chacun ${ }^{30}$. Ainsi elle suppose de promouvoir un accès équitablement partagé de tous aux ressources naturelles et permet de lutter contre l'appropriation de ressources naturelles par quelques-uns au détriment des autres. Cette justice est envisagée selon la conception reformulée de Rawls d'égalité et d'équité, c'est-à-dire que les inégalités ne sont tolérables que si elles profitent à chacun et corrélativement, si leur diminution est susceptible d'aggraver la situation de l'ensemble de la collectivité $^{31}$. On évoque notamment le concept de justice environnementale à propos des peuples autochtones d'Amérique du Sud dans leurs rapports avec les laboratoires pharmaceutiques qui exploitent leurs connaissances des plantes médicinales ${ }^{32}$. Ce rapport déséquilibré a donné lieu en 2010 au Protocole de Nagoya sur le partage équitable des avantages découlant de l'accès aux ressources génétiques $^{33}$, visant ainsi à rééquilibrer le droit d'accès aux ressources et à rétablir une justice.

\section{B. Les approches juridiques revisitées sur lesquelles construire le droit}

On a déjà assisté à une évolution de la théorie juridique, par exemple en termes de droit en réseau ${ }^{34}$, qui a quelque peu ébranlé la perception de l'organisation et du fonctionnement du droit. Selon cette conception, les systèmes juridiques ne sont pas organisés hiérarchiquement, l'Etat n'est plus le seul cadre de référence lorsque l'on veut penser le droit, il n'existe pas un seul ordre juridique mais une multitude de systèmes entretenant entre eux des rapports

\footnotetext{
${ }^{30}$ Consulter : Agnès Michelot (dir.), Equité en environnement. Quel(s) modèle(s) de justice environnementale ?, Bruxelles : Larcier, 2012.

${ }^{31}$ John Rawls, Théorie de la justice, Paris : Points, coll. Points Essais, 2009.

32 Catherine Aubertin, Florence Pinton, Valérie Boisvert, Les Marchés de la Biodiversité, Paris : IRD Editions, 2007.

${ }^{33}$ Protocole de Nagoya sur l'accès aux ressources génétiques et le partage juste et équitable des avantages découlant de leur utilisation du 29 octobre 2010, Secrétariat de la Convention sur la Diversité Biologique, 2012, https://www.cbd.int/abs/doc/protocol/nagoya-protocol-fr.pdf

${ }^{34}$ François Ost, Michel Van de Kerchove, De la pyramide au réseau ? Pour une théorie dialectique du droit, Bruxelles: Publication des Facultés Universitaires Saint-Louis, 2002.
} 
complexes et parfois étranges. En ce sens donc, le droit en réseau renvoie à un droit dont l'agencement n'est pas envisagé à l'image d'une pyramide qui comprend en son sommet la constitution et à sa base le contrat, ainsi que l'avait proposé Kelsen. Le droit en réseau n'envisage pas davantage le droit sous formes des formes binaires entre droit public et droit privé, ou encore entre Civil law et Common law. Le droit en réseau est une organisation des normes juridiques, voire aussi non juridiques, envisagée à l'horizontal, avec des interconnections multiples entre ces normes et entre les acteurs qui les portent (personne publique, privée, individus, scientifiques...) ${ }^{35}$. C'est un droit qui repense a relation entre gouvernants et gouvernés.

Cette conception a trouvé des implications dans la théorie de la validité de la norme juridique, questionnant ainsi la validité associée à la seule légalité des normes ${ }^{36}$. Au sein de la doctrine, on a vu alors nuancer la manière dont une norme acquiert sa validité (par sa légitimité sociale, par son effectivité, par son efficacité... $)^{37}$ et la manière dont une norme conserve ou non sa validité (validité conditionnelle, relative, provisoire, absolue... $)^{38}$. Dès lors, et selon ces approches, les normes juridiques ne sont pas valides parce qu'elles tirent leur légitimité de l'autorité publique qui les a émises ou parce qu'elles sont conformes à la règle juridique qui leur est supérieure, mais elles sont valides parce que, notamment, les destinataires participent à leur élaboration et à leur application, parce qu'elles expriment des valeurs sous-jacentes à la société, et parce que, en participant à l'écriture et à la détermination du contenu de ces normes, les destinataires les acceptent plus facilement et y adhèrent.

Ces approches ont impacté les approches relatives à la

\footnotetext{
35 Ibid.

36 Hans Kelsen, «Quel est le fondement de la validité du droit?», Revue internationale de criminologie, 1956, p. 161.

${ }^{37}$ Pierre Lascoumes, Evelyne Serverin, «Théories et pratiques de l'effectivité du droit », Revue Droit et société, 1986, n² 2, p. 106 ; Maria-Jose Farinas Dulca, «El problema de la validez juridica », Civitas, 1991, p. 135

${ }^{38}$ Adélie Pomade, La Société Civile et le droit de l'environnement. Contribution à la réflexion sur les théoires du droit et de la validité, Paris: LGDJ, 2010 ; Ernesto Garzon Valdes, «Algunos modelos de validez normativa, », Revista latinoamericana de filosofía, 1977, Vol. III, $\mathrm{n}^{\circ}$ 1, p. 59 ; Jean Haesaert, Théorie générale du droit, Bruxelles : Bruylant, 1948, p. 442.
} 
construction du droit en ce qu'elles supposaient déjà une implication plus grande de la société dans sa production. Actuellement, il semble cependant que, de nouveaux, les « faits » conduisent à envisager de nouveaux modèles sur lesquels construire le droit. Pour mieux les comprendre ou à tout le moins, comprendre leur finalité et leur idéologie, on peut partir d'un exemple à travers lequel ces deux théories prennent un sens.

Imaginons une convention conclue entre une entreprise productrice et une coopérative de distribution de miel avec des agriculteurs pour qu'ils plantent des acacias en bordure de leur champ. Cette technique s'appelle l'agro-foresterie. L'objectif est de favoriser le processus de pollinisation et maintenir la production de miel. En contrepartie, l'agriculteur est rémunéré et selon l'évolution de la production (miel d'acacia ou toutes fleurs par exemple), le contrat est réajusté en modifiant la nature des plantations. Les dispositions contractuelles sont déterminées au cas par cas en tenant compte des données sociales, écologiques, géographiques, économiques dans lesquelles le contrat va être mis en œuvre. Ce mécanisme est, de manière simplifiée, celui que l'on appelle «paiement pour services environnementaux » $(\mathrm{PSE})^{39}$. Il a été mis en place notamment pour préserver la biodiversité et les services écosystémiques, c'est-à-dire les bénéfices que l'homme tire du fonctionnement des écosystèmes (stockage de carbone par les forêts, purification de l'eau par les océans...).

En cela donc en pratique, les PSE renvoient à une convention, entre un agriculteur et une personne publique ou privée, ou les deux, qui prévoit une rémunération en contrepartie de pratiques alternatives adoptées dans sa profession pour maintenir un bénéfice que l'homme tire du fonctionnement d'un écosystème. En regardant d'un peu plus près, on retrouve directement l'idée du PSE dans le programme de soutien aux pratiques agroenvironnementales «Prime-vert» adopté par le ministère de l'Agriculture, des Pêcheries et de l'Alimentation au Québec ${ }^{40}$. Ce programme vise en effet à aider les exploitations

\footnotetext{
39 Selon Wunder, les PSE sont des transactions volontaires entre un ou des fournisseurs et bénéficiaires concernant des SE prédéfinis, et qui font l'objet d'ententes conditionnelles.

40 Sophie Lavallée, Jérôme Dupras, «Regard sur les systèmes de paiement pour écosystémiques en milieu agricole au Québec », Développement durable et
} 
agricoles et les autres acteurs de l'industrie bio-alimentaire à relever les défis que représente la protection de l'environnement. Plus largement, cette attention portée aux services écosystémiques se retrouve aussi dans les «fiches de bonnes pratiques » éditées par Santé Canada qui recommandent des «pratiques exemplaires » en matière de pulvérisation de pesticides ou d'utilisation de semences traitées, et ce pour limiter les impacts sur les insectes pollinisateurs.

Ces Paiements se développent à l'échelle mondiale, et se présentent sous des formes très diverses, souvent sans encadrement juridique réellement prédéfini. Il conviendra d'insister aussi sur le fait qu'ils sont des instruments dits «basés sur le marché de la biodiversité ${ }^{41}$ ou des instruments économiques à l'origine façonnés par la société. Ils ne sont donc pas un produit du droit, mais l'attention des juristes à leur égard laisse entrevoir la possibilité d'une évolution possible de la norme juridique et de sa construction. En effet, par leur flexibilité et adaptabilité, ces instruments suggèrent ou dessinent de nouvelles approches théoriques. Deux théories en émergence peuvent donc être ici évoquées : l'adaptive Law et la logique graduée.

L'adaptive Law de l'américain Graig Arnold, consiste à intégrer diverses méthodes dans le processus de fabrication du droit pour qu'il soit en adéquation avec la complexité du vivant qu'il doit encadrer. Sans trop rentrer les détails de cette approche, il convient cependant d'attirer l'attention sur ces méthodes pour saisir la manière dont elles résonnent avec le mécanisme de PSE. Tout d'abord, cette théorie suppose la prise en compte de plusieurs acteurs, modes d'actions, d'échelles, ensuite elle postule une adaptation du droit à la flexibilité contextuelle et enfin, elle soutient l'intégration de processus récursifs ${ }^{42}$.

Par ailleurs, si l'on prend de la hauteur sur la construction du droit à partir de cet adaptive Law, on remarque la différence de conception du droit qu'elle peut engendrer par rapport à une

territoires, 2016, Vol. 17, https://isfort.uqo.ca/sites/isfort.uqo.ca/files/fichiers/public ations_ISFORT/lavallee_dupras_2016_developpementdurable.pdf

${ }^{41}$ Romain Pirard, Emma Broughton, "What's in a name? Market-based Instruments for Biodiversity", Policy brief IDDRI, 2011.

42 Craig Anthony Arnold, Lance H. Gunderson, "Adaptive Law and Resilience", Legal Studies Research Paper Series, University of Louisville, 2014, nº4. 
approche classique. Le tableau ci-dessous met en lumière ces différences $^{43}$ :

TABLEAU - différence entre Adaptive Law et Maladaptive Law

\begin{tabular}{|c|c|c|}
\hline & $\begin{array}{l}\text { Droit maladapté/maladaptive } \\
\text { Law }\end{array}$ & Droit adapté/adaptive Law \\
\hline Objectifs & $\begin{array}{l}\text { Les régimes juridiques visent à } \\
\text { garantir une stabilité absolue } \\
\text { d'un seul système : politique ou } \\
\text { économique ou écologique. }\end{array}$ & $\begin{array}{l}\text { Les régimes juridiques visent de } \\
\text { multiples formes de résilience: } \\
\text { l'adaptabilité réciproque du } \\
\text { systèmes social et écologique } \\
\text { (équilibre des socio-écosystèmes), } \\
\text { en intégrant les éléments } \\
\text { constitutifs des sur-systèmes } \\
\text { comme les institutions et les } \\
\text { communautés. }\end{array}$ \\
\hline Structure & $\begin{array}{l}\text { Le droit est mono-centré, } \\
\text { d'utilisation fragmentée et } \\
\text { fourni des réponses uni-modales } \\
\text { pour résoudre un problème. }\end{array}$ & $\begin{array}{l}\text { Le droit est polycentrique, } \\
\text { utilisant des réponses multi- } \\
\text { modales et multi-scalaires. Il } \\
\text { s'agit donc de prendre en } \\
\text { compte différentes échelles pour } \\
\text { résoudre des problèmes. }\end{array}$ \\
\hline Méth & $\begin{array}{l}\text { Le droit contrôle la société au } \\
\text { travers de règles, de limitations } \\
\text { aux actions, de demande de } \\
\text { certitudes et d'abstractions } \\
\text { juridiques qui résistent au } \\
\text { changement. }\end{array}$ & $\begin{array}{l}\text { Le droit facilite la résilience } \\
\text { sociale et écologique au travers } \\
\text { de processus adaptatifs adaptés } \\
\text { aux conditions changeantes, au } \\
\text { travers de normes établies à } \\
\text { partir du contexte, au travers de } \\
\text { tolérance à l'incertitude, et au } \\
\text { travers de prise de décision } \\
\text { flexible }\end{array}$ \\
\hline Processus & $\begin{array}{l}\text { Le droit suppose une prise de } \\
\text { décision rationnelle et linéaire, } \\
\text { et son implémentation résulte } \\
\text { d'une seule autorité habilité et } \\
\text { centralisée. }\end{array}$ & $\begin{array}{l}\text { Le droit admet et adopte des } \\
\text { processus itératifs qui supposent } \\
\text { des feedback et des boucles } \\
\text { récursives entre une pluralité de } \\
\text { participants, dont bien sûr les } \\
\text { acteurs sociétaux. }\end{array}$ \\
\hline
\end{tabular}

On comprend alors mieux comment ces PSE, à l'origine instruments créés par la société, en viennent à inspirer les théoriciens du droit en faveur d'une reconstruction de la méthode même de construction du droit.

${ }^{43}$ Ce tableau, traduit par l'auteur de l'article, est tiré de : Craig Anthony Arnold, Lance H. Gunderson, «Adaptive Law and Resilience », précit. 
La seconde théorie que l'on peut évoquer est la logique graduée. Elle constitue une réflexion plus prospective ${ }^{44}$. L'idée est d'envisager la régulation produite par les acteurs étatiques et non étatiques non pas de manières exclusive ou complémentaire, mais de manière équilibrée. Cette logique implique de répondre à deux questions principales : 1/ la régulation d'une situation par des acteurs privés est-elle effective et efficace ? 2/ A défaut d'une régulation effective et efficace, jusqu'à quel point la régulation étatique doitelle intervenir pour garantir l'une et l'autre, sans démobiliser les acteurs impliqués en leur donnant un sentiment de contrainte ou d'imposition?

Si l'on consent à entrer davantage dans cette théorie, on peut envisager qu'adopter ce questionnement revient à accepter de poser l'hypothèse de l'inutilité de l'intervention du droit si les acteurs autorégulent les pratiques de manière suffisamment efficaces pour maintenir ou restaurer les services. Dans l'exemple illustratif des PSE, il s'agirait du service de pollinisation.

La finalité d'une telle approche consiste donc pour le droit à intervenir sur la régulation en place de manière équilibrée et au plus près de la réalité pour ne pas se déconnecter des besoins locaux ou des contextes. L'originalité de cette approche réside donc dans la flexibilité du droit à l'égard des socio-écosystèmes, mais elle réside aussi dans son adaptabilité car elle ne consiste pas à intervenir de manière systématique, unilatérale et générale en imposant par exemple un décret ou un arrêté encadrant, interdisant ou réglementant, mais à intervenir sur la régulation mise en place de manière circonstanciée, par des instruments juridiques par exemple négociés (dont la nature reste encore à déterminer), pour permettre aux acteurs concernés de tirer bénéfice d'un service tout en favorisant sa pérennité ${ }^{45}$.

${ }^{44}$ Adélie Pomade, Charles-Hubert Born, Regulation and Market-Based Instruments for Biodiversity and Ecosystem Services. The Cases of Payment for Ecosystem Services and Habitat Banking Scheme. Biodiversity and ecosystem services overview, Report, INVALUABLE "Valuations, Markets and Policies for Biodiversity and Ecosystem Services" ( $7^{\mathrm{e}}$ PCRD - Biodiversa), 2015, Laboratoire SERES, Université Catholique de Louvain (Belgique).

${ }^{45}$ Adélie Pomade, «Les paiements pour services environnementaux contribuent-ils à l'émergence d'un « gradient de juridicité »? », Revue Vertigo, 2016, en ligne : https://vertigo.revues.org/17084 
Pour reprendre l'exemple du recours à l'agro-foresterie, dans l'hypothèse où la régulation mise en place par les agriculteurs se montrerait inefficace pour préserver le service de pollinisation, l'autorité publique pourrait envisager de négocier avec eux une diminution de leur utilisation de pesticides, en cherchant des solutions efficaces afin que cette diminution éventuelle ne préjudicie pas leur récolte (par exemple, une quantité plus faible de rendement mais une meilleure qualité et donc un prix de vente revu à la hausse). On pourrait même envisager que le droit acquiert ici une nouvelle fonction : une fonction d'assistance. En ce sens, il pallierait les insuffisances de la régulation sociale et interviendrait pour garantir une efficacité et une effectivité normative.

A bien y regarder, ces nouveaux modèles d'adaptive Law ou de logique graduée semblent rendre compte du passage d'une approche autopoḯtique du droit dont parlent notamment Teubner et Luhmann, à ce que l'on appelle une approche intégrée, qui va au-delà de ce que Carbonnier appelait internormativité.

En effet, en droit, on parle d'approche autopoiétique. Conceptualisée en sociologie par Luhmann ${ }^{46}$ et reprise en droit par Teubner $^{47}$, puis en droit de l'environnement ${ }^{48}$, cette vision considère que le droit s'ouvre aux autres disciplines pour récupérer des informations (données non juridiques), mais se referme ensuite sur lui-même pour traiter les informations recueillies avec ses propres catégories et concepts. Ainsi, le droit s'ouvre pour accueillir des données qu'il expose à un jeu d'exclusions et de hiérarchisations, sans laisser place à la création d'interrelations. Cette perspective entrave toute porosité du droit à l'égard d'autres disciplines.

Le Doyen Jean Carbonnier qui dénonçait déjà en son temps cette étanchéité du droit a développé un concept toujours fortement d'actualité : l'internormativitée ${ }^{49}$. Ce concept a d'ailleurs été repris et

46 Niklas Luhmann, «Le droit comme système social », Droit et société, 1989, p. 53-66.

47 Gunther Teubner, Le droit, un système autopoïétique, Paris : Presses Universitaires de France, 1993.

48 Centre d'analyse stratégique, Approche économique de la biodiversité et des services liés aux écosystèmes. Contribution à la décision publique, Rapport, Centre d'analyse stratégique, 2009.

49 Jean Carbonnier, «Les phénomènes d'internormativité », European yearbook in the law and sociology, La Hague, M. Nijhoff, 1977. 
exploré par nombre d'auteurs. L'internormativité s'entend de deux sens différents mais ces deux sens sont de plus en plus présents en droit. Le premier, à l'origine plus juridique donc, évoque le passage d'une norme ou d'une règle d'un ordre normatif à un autre ${ }^{50}$. Il s'agit par exemple de la reprise d'une norme technique ou scientifique comme une expertise dans une norme juridique. Le second, à l'origine plus sociologique, envisage les contacts et les rapports de pouvoir, d'influence ou d'interaction qui peuvent être observés entre deux ou plusieurs de ces systèmes $^{51}$. Dans ce cas, n'est pas concerné le passage d'une norme d'un système à l'autre mais plutôt l'ensemble des échanges entre les acteurs des systèmes. C'est par exemple, dans le cadre des processus de négociation d'un traité international, tout ce qui renvoie aux stratégies de séduction mises en place par les lobbies, ou encore aux campagnes de sensibilisation de la vulnérabilité des peuples autochtones face à l'érosion de la biodiversité et au changement climatique. Si les sciences sociales s'intéressent à cette seconde signification, les juristes en revanche n'envisagent souvent que la première, de surcroît dans un sens assez restrictif.

Toutefois, on voit se développer actuellement ce que l'on appelle des approches intégrées, c'est-à-dire des approches qui consistent à adopter une méthode d'analyse pluridisciplinaire pour examiner un objet sous plusieurs angles ${ }^{52}$. Ces approches mobilisent, ensemble, géographie, écologie, sociologie ou encore économie, pour comprendre un mécanisme, un objet ou un processus faisant l'objet d'un enjeu sociétal. On rencontre cette approche intégrée lorsque l'on parle par exemple de la "gestion intégrée des zones côtières ». En pratique, il s'agit une démarche de gouvernance des territoires littoraux prenant simultanément en compte les enjeux terrestres et marins, naturels économiques et sociaux d'une zone littorale définie comme territoire cohérent de réflexion et d'action.

50 Karim Benyekhlef, Une possible histoire de la norme. Les normativités émergentes de la mondialisation, Montréal : Thémis, 2008.

${ }^{51}$ Guy Rocher, «Les phénomènes d'internormativité : faits et obstacles », In J.G. Belley (dir.), Le droit soluble. Contributions québécoises à l'étude de l'internormativité, Paris : LGDJ, 1996, p. 27.

52 Mathieu Doat, «Gestion intégrée des zones côtières et décentralisation », VertigO, Hors-série 18, Décembre 2013, en ligne : https://vertigo.revues.org/14268 
Toutefois, il est encore difficile d'adopter cette approche systémique lors de la construction du droit, notamment par sa tendance à son étanchéité.

\section{LA CONSTRUCTION DU DROIT A PARTIR DE L'EXPRESSION CITOYENNE}

La construction du droit à partir de l'expérience citoyenne peut se décliner sur deux plans: le droit positif (A) et la pratique (B).

\section{A. La revendication des droits fondamentaux dans la construction du droit}

Le droit à l'information, le droit à l'éducation, la nondiscrimination, le droit à participer aux processus décisionnels, ou encore le droit de propriété sont autant de droits fondamentaux et de libertés fondamentales reconnus par les systèmes juridiques internationaux, régionaux et nationaux. Autant de droits et de libertés mis en œuvre ou garantis par des instances spécifiques, comme la Cour européenne des droits de l'homme, en Europe. Mais à bien regarder, l'exercice ou l'évocation de ces droits et libertés rend possible la construction du Droit au travers de plusieurs canaux:

En premier lieu, la construction du droit à partir de la revendication de ces droits s'observe à travers la jurisprudence, lorsque des demandeurs dénoncent la lésion d'un droit fondamental. Dans ce cas, l'invocation de la violation d'un droit peut conduire les juges à exiger qu'en certains cas, des mesures légales soient prises.

On évoquera par exemple l'affaire Brincat et autres $c$. Malte jugée par la Cour européenne des droits de l'Homme en $2014^{53}$. Cette affaire concernait des ouvriers de chantier naval qui avaient été exposés à l'amiante pendant plusieurs décennies des années $1950 \mathrm{au}$ début des années 2000 et qui en avaient gardé des séquelles. Les requérants se plaignaient en particulier d'avoir été exposés à l'amiante, ou que leurs proches aient été exposés à l'amiante, et reprochaient au gouvernement maltais de ne pas les avoir protégés,

53 CEDH, 24 juillet 2014, Brincat et autres c. Malte, file:///C:/Users/ad/Downloads/003-4833696-5896983.pdf 
ou de ne pas avoir protégé leurs proches, des conséquences dramatiques de cette exposition. La Cour a conclu à la violation de l'article 2 de la Convention consacrant le droit à la vie à l'égard des requérants dont le proche était décédé, et à la violation de l'article 8 de la Convention relatif au droit au respect de la vie privée et familiale, à l'égard des autres requérants. Elle a notamment jugé que, étant donné la gravité des risques liés à l'amiante, même si les Etats ont une certaine marge d'appréciation pour décider comment gérer de tels risques, le gouvernement maltais avait manqué aux obligations positives que lui impose la Convention, en ce qu'il n'avait pas légiféré ni pris de mesures pratiques ou réglementaires pour faire en sorte que les requérants soient suffisamment protégés et informés du risque auquel étaient exposées leur santé et leur vie. Estimant que, depuis le début des années 1970 au plus tard, le gouvernement maltais savait ou aurait dû savoir que la santé des ouvriers du chantier naval était mise en danger par l'exposition à l'amiante, la Cour a observé qu'il n'avait pas pris de mesures positives pour parer à ce risque avant 2003. La revendication des droits fondamentaux lésés fait apparaître ici des lacunes dans la prise de mesures juridiques et laisse donc présumer que cette décision permettra à l'avenir de contraindre les Etats placés dans une situation similaire à construire du droit pour protéger ses ressortissants.

L'invocation de la lésion d'un droit fondamental peut aussi avoir pour objectif la revendication de la nécessité de «construire » un autre droit fondamental. On évoquera brièvement à cet égard l'affaire Sarnia's Chemical Valley dans laquelle une décision du ministre de l'environnement de l'Ontario autorisait l'entreprise Suncor Energy Product à accroître une partie de la production de sa raffinerie. Cette décision était considérée par les communautés résidant à proximité comme violant leurs droits fondamentaux garantis par la Charte canadienne des droits et libertés fondamentales, précisément, les articles 7 et 15 relatifs au droit à la vie, à la liberté, à la sécurité de la personne et à l'égalité. L'un des objectifs était de faire annuler cette autorisation et ainsi de contribuer à faire reconnaître de nouveaux droits comme, le droit à un environnement sain, l'objectif était donc de contribuer à construire ce droit fondamental.

En second lieu, la construction du droit à partir de l'exercice 
des droits fondamentaux s'observe à travers la décision publique, lorsque les citoyens participent au processus de prise de décision. Dans ce cas, plusieurs notions sont mobilisées: les notions de processus discursifs ${ }^{54}$, de droit négocié ${ }^{55}$, de procéduralisation ${ }^{56}$, mais encore les notions d'internormatitivité dans son sens sociologique d'inter-influence entre les acteurs, les notions construction conjointe ou co-construction de la norme juridique, ou encore de gouvernance réflexive ${ }^{57}$.

Pour illustrer ce domaine de la décision publique, on mentionnera l'expérience belge appelée "Gant en garde ». Il s'agit d'une initiative de la ville de Gant visant un double objectif: d'une part, faire participer le public à l'évolution des modes de consommation de la nourriture et d'autre part, faire prendre conscience de l'impact de la consommation de nourriture sur le climat. Pour cela, la ville de Gant a mis en place de nombreux marchés en ville et dans sa périphérie, afin de favoriser les circuits courts (producteur-consommateurs) et d'informer sur les différentes qualités de produit (agriculture biologique ou conventionnelle, nourriture carnivore, végétarienne et végétalienne). Par ailleurs, pour prendre ses décisions, la ville consulte deux types de personnalités. D'un côté, des personnalités «qualifiées » regroupées au sein du Comité régional de distribution de la nourriture, comme des représentants d'agriculteurs et d'associations, des citoyens éclairés. D'un autre côté, des personnes qui se sentent concernées. Ces denrières participent par l'intermédiaire de forums publics ou de forums en ligne qui permettent à tous de partager des informations.

Au-delà de la dimension démocratique qui transforme un consommateur en co-inventeur de solutions ${ }^{58}$, cette initiative illustre :

\footnotetext{
${ }^{54}$ Marc Maesschalk, «La participation des groupes d'intérêt dans la «nouvelle gouvernance européenne ». Une nouvelle forme de biopouvoir?», Revue française d'histoire des idées politiques, 2016, $\mathrm{n}^{\circ}$ 1, p. 185-204.

${ }^{55}$ Pierres Lascoumes, «Négocier le droit, formes et conditions d'une activité gouvernementale conventionnelle», Politique et management public, 1993, p. 47-83.

${ }^{56}$ Jurgen Habermas, De l'éthique de la discussion, précit.

57 Tom Dedeurwaerdere, «Bioprospection, gouvernance de la biodiversité et mondialisation ", Cahiers du CPDR, 2004, en ligne : https://perso.uclouvain.be/tom.dedeurwaerdere/articles\%20Tom/dedeurwaerdere104.pdf

${ }^{58}$ Rob Hopkins, Ils changent le monde !, Paris : Editions du Seuil, 2014.
} 
- Comment l'autorité publique construit le droit en rendant ses décisions par la consultation d'un public concerné.

- Comment la société civile utilise et revendique ses droits fondamentaux, comme le droit à l'information, le droit de participer ou le droit de consommer de la nourriture saine, pour contribuer à construire le droit, à construire les décisions publiques.

- Comment ces droits fondamentaux sont liés avec la notion de responsabilité et dès lors, participent à la réécriture du droit de la responsabilité. En effet, selon ce cas d'étude de Gant, les citoyens ont le droit d'avoir accès à la nourriture, mais ce droit doit être reconsidéré et repensé en raison de son impact sur le climat. Ainsi, sont évoquées plusieurs implications selon les modes de consommation :

○ Consommer des produits issus de l'agriculture conventionnelle n'est pas sans lien avec l'impact de l'épandage des engrais azotés minéraux et organiques car cela conduit à une émission de protoxyde d'azote au pouvoir réchauffant 310 fois plus grand que le $\mathrm{CO} 2$.

- Ne pas consommer local favorise les importations.

- Consommer de la viande animale en grande quantité favorise la production de méthane provenant des déjections animales, 28 fois plus "réchauffant » que le dioxyde de carbone ( $\mathrm{CO} 2)$.

Plus loin, ces droits fondamentaux, socle sur lequel le droit est construit, mettent en lumière des enjeux délicats. Le premier enjeu est la légitimité de la norme juridique. Cela invite à se poser la question de «qui détient les clefs de l'élaboration du droit? ». Ce questionnement peut conduire à mettre de la distance avec la théorie de la légitimité fondée sur la légalité des normes. Le deuxième enjeu est la prise en considération du droit comme un «instrument» agit par la société. En ce sens, la société civile peut construire le droit en utilisant ses droits comme des instruments ${ }^{59}$. Un troisième enjeu

59 Guy Rocher, » Droit, pouvoir et domination », Sociologies et Sociétés, 1986, p. 33-46. 
renvoie au fait que les droits fondamentaux exercés notamment durant le processus de prise de décision, conduisent à questionner les notions de citoyenneté, de démocratie et de gouvernance.

\section{B. L'utilisation des savoirs et des expériences de la société dans la construction du droit}

A la fin du XXe siècle, nous avons assisté à la remise en cause de la prééminence de l'expertise scientifique sur la décision publique. Cela est dû entre autres à la défaillance des experts à propos de certains phénomènes qui ont causé de sérieuses conséquences. L'encéphalopathie spongiforme bovine, plus connue sous le nom de la maladie de la vache folle, et avant elle, le virus de l'immunodéficience humaine (VIH), connu sous le terme de SIDA, constituent deux exemples prégnants.

Cette crise de la rationalité scientifique a affecté la relation entre science et société ${ }^{60}$. Petit à petit, les expertises scientifiques ont été mises en question par les associations estimant que les citoyens pouvaient finalement être eux aussi des co-experts capables de produire des savoirs à partir de leurs expériences.

Quel lien tisser entre cette remise en cause et la construction du droit? Il convient de garder à l'esprit que l'expertise scientifique est considérée comme une source d'élaboration du droit: un expertise sur le climat peut alerter d'un phénomène de réchauffement global et motiver l'introduction de quotas d'émission ou de diverses obligations dans les traités internationaux et les réglementations européennes. Une ouverture de l'expertise aux citoyens demandée par les groupements associatifs devient alors intéressante dans le processus de construction du droit parce qu'elle implique une mobilisation conjointe de l'expertise scientifique avec l'expertise citoyenne, ce qu'Edgar Morin appelle la «reliance des savoirs ${ }^{61}$. La rencontre de ces deux types de savoirs et leur mise en dialogue, permet la formulation de théories, de scénarios, d'hypothèses scientifiques, qui vont nourrir la construction du droit. L'accent est

60 Céline Granjou, «L'expertise scientifique à destination politique », Cahiers internationaux de sociologie, 2003, p. 175-183.

${ }^{61}$ Edgar Morin, La Méthode. L'Ethique, 2004, Paris : Editions du Seuil, p. 269. 
alors placé sur la construction du droit à partir du terrain.

Il existe actuellement deux outils de mobilisation du savoir qui influencent la construction du droit. Le premier est la citizen science, très utilisée en sociologie ${ }^{62}$. Cet outil consiste dans la mise en place de forums ou groupes de discussion où les participants partagent leurs savoirs acquis par expérience. Les premiers groupes du genre ont été mis en place en médecine, notamment justement pour les malades victimes du VIH, et ce en visant plusieurs objectifs: que les malades expliquent leur ressenti sur la maladie à différents stades de son évolution, qu'ils rendent compte de leurs vies sociale, familiale, parfois professionnelle, une fois la maladie déclarée. L'objectif était de recueillir leur expérience afin de trouver des solutions adaptées et applicables aux autres malades placés dans la même situation qu'eux, d'atténuer leurs souffrances, leur mal-être, ou encore, de reproduire ou s'inspirer des démarches qu'ils auraient adoptées spontanément pour les appliquer aux autres patients. Un autre exemple, en environnement cette fois, renvoie à la mise en place en France de workshops sur les pratiques agricoles. Ces workshops collectent les expériences des fermiers et agriculteurs. Certaines déclarations sur la relation entre la maladie de Parkinson et les pesticides, ajoutées aux travaux scientifiques en cours, ont influencé le législateur dans sa reconnaissance en 2012 de cette maladie comme d'une "maladie professionnelle" dans le secteur agricole ${ }^{63}$.

Le second outil de mobilisation du savoir qui influence la construction du droit est la science participative. Cette technique est davantage appropriée par l'écologie ${ }^{64}$. Elle renvoie à la participation des individus à la science par l'intermédiaire de leurs observations. En France par exemple, le Muséum National d'Histoire Naturelle a mis en place un programme d'observation des insectes qui a pour but d'obtenir des données quantitatives sur les insectes pollinisateurs et floricoles en France métropolitaine en mesurant les variations de leur

62 Florian Charvolin, «Le Programme Federwatch et la politique des grands nombres », Développement durable et territoires, 2004, http://developpementdurable.revues.org/687

63 Décret $\mathrm{n}^{\circ}$ 2012-665 du 4 mai 2012 révisant et complétant les tableaux des maladies professionnelles en agriculture, JO du 6 mai 2012.

64 Anne Tessèdre, Denis Couvet, «Science participative», Société française d'écologie, 2011, https://www.sfecologie.org/regard/regards-r11-teyssedre-et-couvet/ 
diversité et la variation de la structure des réseaux de pollinisation ${ }^{65}$. D'autres initiatives sont menées également par des structures maritimes cette fois, comme des Aquarium ou encore des associations de protection de l'environnement, qui lancent des programmes d'observation des mammifères marins ou des programmes d'observation de la faune et de la flore littorales. Une fois que ces «données d'opportunité », c'est ainsi qu'on les appelle, sont collectées par les individus profanes, elles sont transmises aux scientifiques. Plusieurs outils sont mis à disposition des individus pour réaliser cette transmission. Ce peut être par courrier, par mail, voire par l'intermédiaire d'applications téléchargeables en ligne dédiées aux observations, par lesquelles on peut, grâce à son smartphone, envoyer la photographie de son observation en même temps que son commentaire. Cette co-construction des enjeux scientifiques questionne les écologues eux-mêmes sur l'impact de cette expertise co-créée sur l'élaboration de la norme juridique et sur l'élaboration des politiques publiques ${ }^{66}$. En ce sens, toutes ces données pourraient peut-être constituer ce que les écologues appellent des indicateurs de biodiversité, c'est-à-dire des résumés d'information sur l'état moyen de la biodiversité dans certaines conditions à un instant donné, qui seraient alors de précieux outils de réflexion et de concertation pour les acteurs, décideurs et gestionnaires de la biodiversité.

Cette forme d'engagement des individus dans la construction du droit affecte les enjeux d'effectivité et d'efficacité de la norme juridique parce qu'elle promeut d'une part, une traduction du savoir citoyen en termes techniques et d'autre part, une vulgarisation du savoir scientifique. On observe ainsi une compréhension réciproque du champ linguistique. La norme est alors mieux acceptée par ses destinataires, elle répond de manière plus cohérente aux besoins sociaux, économiques, environnementaux et culturels. Ces nouveaux canaux de participation impactent finalement directement sur l'enjeu principal du droit : son adéquation avec les réalités sociétales.

${ }^{65}$ Programme Spipoll-Vigie Nature, http://www.spipoll.org/

66 Denis Couvet, Anne Tessèdre, «Sciences participatives et biodiversité : de l'exploration à la transformation des socio-écosystèmes », Cahier des Amériques latines, 2013, p. 49-64. 
En guise de conclusion, on peut ajouter que ces quelques propos ont mis en lumière un droit en mouvement, un droit en changement, non pas tant dans sa forme ou ses outils, que dans sa fabrication et sa fonction. Le droit ainsi produit par la société ou à l'inverse, producteur de repères et d'assistance pour la société entraîne alors une reconfiguration du rôle de l'autorité publique, du rôle du l'Etat dans sa mission de régulation des comportements humains. Il semble que l'on assiste à l'avènement de nouveaux modèles de régulation sociale et politique qui sont issus d'une réappropriation de la chose juridique, de la chose publique, par nos sociétés contemporaines. On se permettra de terminer sur une citation du dernier ouvrage de Jacques Commaille qui peut être lu à la fois comme un miroir de ce qui vient d'être présenté aujourd'hui et comme une ouverture à ces propos : "l'enjeu est désormais de saisir les causes, les manifestations, les devenirs possibles d'une régulation polycentrique de par la multiplicité de ses polarités, de ses ancrages géographiques et culturels. Ce régime de régulation s'établit en référence à des pôles (politique, culturel, social, économique), le juridique était lui-même partie prenante de cette configuration ${ }^{67}$.

${ }^{67}$ Jacques Commaille, A quoi nous sert le droit ? précit. 
